\title{
Invited review
}

\section{Advances in echocardiography: global longitudinal strain, intra-cardiac multidirectional flow imaging and automated $3 \mathrm{~d}$ volume analysis}

Oben Baysan, Ezgi Polat Ocakli, Yasemin Saglam, Tugba Kayhan Altuner

Guven Hospital, Ankara, Turkey

\begin{abstract}
Echocardiography has the long development history beginning with amplitude imaging. Nowadays, two- and threedimensional imaging are standard tools available in almost every echocardiography machine. Myocardial deformation imaging is gaining popularity out of research projects. The future will bring new and sophisticated tools for echocardiographic analysis such intracardiac flow imaging and an automated 3D volume calculation.
\end{abstract}

Key words: global longitudinal strain, left atrial strain, intracardiac flow imaging, automated 3D volume analysis

(Heart Vessels and Transplantation 2018; 2: doi: 10.24969/hvt.2018.83)

\section{Introduction}

Echocardiography has been evolved from plain amplitude imaging (A-Mode) to sophisticated threedimensional acquisition of the whole heart in a single heartbeat. In 1954 Dr Edler wanted to identify patients with severe mitral regurgitation. He, together with $\mathrm{Dr}$. Hertz, succeeded to find a method we called it as MMode echocardiography (1). Together with developments in engineering, computer hardware and software, we have many echocardiographic tools available for clinical use. Today, M-Mode, 2-dimensional (2D) imaging methods together with pulse-wave and continuous-wave Doppler are standard modalities for any echocardiography machine.

Myocardial deformation imaging carries echocardiographic information to a new level. Different vendors quickly incorporated new methods for assessing myocardial deformation such as tissue Doppler imaging (TDI), speckle tracking echocardiography (STE) and velocity vector imaging. Strain analysis based on TDI or STE has become a reality for daily clinical use. The development of transducers capable of three- dimensional imaging heralded the beginning a new era. Three-dimensional echocardiography (3DE) has gained ground in different clinical scenarios including ventricular mass or volume measurement and strain calculation. 3DE has also been used in interventional procedures for pre-procedural planning, peri-procedural guidance and post-procedural follow-up. Automatic 3D volume analysis will comes with new research possibilities. Adding multidimensional intracardiac flow data to cardiac deformation can yield conceptually better understanding of cardiac dynamics. In this review, we aimed to attract interested readers' attention to these relatively new echocardiographic methods.

\section{Myocardial Deformation Imaging}

Left ventricular global strain (GLS)

Left ventricular ejection fraction (LVEF) is accepted traditionally as a good marker for left ventricular systolic function and has been thought as a strong parameter reflecting contractility. Different ejection fraction (EF) threshold values have been used in decision-making process in patients with heart failure or valvular heart disease. Basically, EF is a simple ratio which is stroke volume divided by left ventricular enddiastolic volume. Unfortunately, it is affected by both loading conditions (preload and afterload) and ventricular end-diastolic volume changes resulting from geometric remodeling (2). Konstam and Abboud recommended that LVEF should not be used for surrogate marker for left ventricular contractility (2). Low sensitivity for revealing subclinical myocardial damage, poor reproducibility values and relatively large intra- and inter-observer variability seem to be other barriers for clinical use of LVEF. More sensitive and reproducible parameters are needed to detect the early adverse myocardial changes in various disease states.

Address for correspondence: Oben Baysan, Guven Hospital Cardiology Department, Simsek Street No: 29 Kavaklidere, Ankara, Turkey, Email: obbaysan@gmail.com, phone number: +90 3124572488

Received: 07.10.2018 Accepted: 08.10.2018 
The heart undergoes constant deformation in each cardiac cycle as shortening-lengthening and rotation around its long axis. Myocardial deformation adds another dimension to cardiac function analysis and deals with the change in myocardial fiber's dimension during cardiac contraction and relaxation. Myocardial fibers have special architecture composed of superficial subepicardial, middle circumferential and deep subendocardial orientation. Those three bundles show deformation in longitudinal, circumferential and radial directions. Strain describes the percentage of deformation in a myocardial fiber compared to its initial length (Fig. 1).

$$
\text { Strain }(\varepsilon)=\frac{\Delta \mathrm{L}}{\mathrm{L}_{0}}
$$

\section{$\mathrm{L}_{0}$ : the original length (grey bar in figure), $\Delta \mathrm{L}$ the change in length (orange bar)}

\section{Figure 1. Definition of strain}

Myocardial velocity data obtained from TDI is used for strain and strain rate measurements (3). TDI can provide us only one-dimensional strain values (longitudinal or transverse). Its angle dependency and low signal to noise ratio have restricted the use of TDI strain.

STE is a technique based on the analysis of speckle's motion. Speckles created by ultrasound wavemyocardium interactions (reflection or scattering) followed by vendor specific algorithm's during cardiac cycle on frame-by-frame basis. Positive values in STE points to the lengthening, thickening or clockwise rotation whereas negative values are reserved for the shortening, thinning or counterclockwise rotation. STE directly tracks myocardium, and hence, permits a better differentiation between the active myocardial segmental deformation and the passive displacement of target segments caused by tethering or global cardiac motion (4). STE based strain measurements have a good correlation with tagged magnetic resonance imaging
(MRI) (5). There had been a hope that STE based deformation imaging would be better suited for revealing segmental dysfunction but subsequent studies have failed to confirm this hypothesis because of high noise in regional deformation parameters. Conversely, a global parameter, GLS, has emerged as a reliable systolic function measurement alternative.

Apical echocardiographic windows are used for GLS calculation because they provide more robust and reproducible images compared to short axis windows. GLS is an early marker of left ventricular dysfunction irrespective of EF values in various diseases including stable coronary artery disease, diabetes and atrial fibrillation (6-8). It has high precision values even among echocardiographers with no experience in strain imaging (intraclass correlation coefficient 0.976 similar to that of expert readers 0.996) (9). Unfortunately, radial or circumferential strain values are not reproducible enough to be used in an echocardiography laboratory. 


\section{How to Measure GLS}

GLS measurement begins with acquiring images with clearly visible endocardial border throughout the whole cardiac cycle. Tracking quality will be higher in these images. The operator should be sure about correct positioning of apex and mitral annulus. Marking mitral annulus at the left atrial side or inappropriate positioning of the sample volume at the left ventricular outflow tract should be avoided. Inclusions of irrelevant anatomic structures such as pericardium or papillary muscles have an impact on resulting GLS values. Step by step approach to GLS measurement and resulting GLS graph from apical four chamber view was depicted in Figure 2 (10).

\section{GLS Normal Values}

According to a meta-analysis GLS values changed from $-15.9 \%$ to $-22.1 \%$ (11) but a value above $20 \% \pm 2 \%$ SD is generally accepted as normal (12). Smiseth et al. proposed that a GLS value $>-12 \%$ (less negative values) indicates severe systolic dysfunction or adverse prognosis; whereas a value $>-15-16 \%$ indicates risk in patients with preserved LVEF.

GLS Intervendor Differences

Farsalinos et al. in The EACVI/ASE Inter-Vendor Comparison Study showed an absolute difference between vendors for GLS was significantly different. Intervendor variability reached up to $3.7 \%$ strain units (13). The interobserver relative mean errors were $5.4 \%$ to $8.6 \%$ for GLS and the intraobserver relative mean errors were $4.9 \%$ to $7.3 \%$. These errors were lower than that for left ventricular ejection fraction and most of the other conventional echocardiographic parameters (13). They concluded that significant inter-vendor differences should be taken into account especially in serial measurements.

\section{GLS in Chemotherapy-Related Cardiac Dysfunction}

Any cancer patient with symptoms of heart failure is considered to have a chemotherapy-related cardiac dysfunction (CTRCD) if their baseline EF value drops more than $5 \%$ points to below $53 \%$ during followup. More than 10 points reduction is required for the CTRCD diagnosis in an asymptomatic patient (14). Unfortunately, EF is an imperfect imaging modality for determination of cardiac toxicity. It is insensitive to early changes in cardiac contractility (15). A decrease in longitudinal shortening compensated by an increase in circumferential shortening, therefore, EF value stays at almost same level even in later stages of cardiotoxicity (16). Fortunately, GLS has been found to be a more sensitive parameter for detecting cardiac toxicity. It has lower intra-observer and inter-observer variability (17). An $11 \%$ reduction in $\Delta$ GLS has a sensitivity of $65 \%$ and a specificity of $94 \%$ for subsequent cardiotoxicity during chemotherapy (18). Negishi et al have proposed a following classification for GLS values in the follow-up of the cancer patients receiving chemotherapy : $<16 \%$ as abnormal, 16-18 borderline, and $>18$ as normal (19).

\section{Left Atrial Strain}

Speckle derived left atrial strain provides more in-depth information about left atrial properties compared to the direct measurement of its anteroposterior diameter or area-length derived volumes. Speckle tracking is somewhat difficult within the thin walled left atrium compared to LV, nevertheless, reservoir-conduit and atrial booster pump phases can be measured by left atrial strain analysis if the QRS complex is taken as reference point (20) (Fig. 3). Apical 4- and 2- chamber views are used for this measurement. First, left atrial endocardium is traced and then the region of interest (ROI) is adjusted according to left atrial wall thickness. Endocardial continuity at the orifices of pulmonary veins and the left atrial appendage is manually adjusted by the operator. The software divides ROI to 12 segments ( 6 for apical 4chamber and another 6 for 2-chamber view) and calculates regional and global left atrial strain values. Normal values for left atrial strain in its reservoir, conduit and atrial contraction phases are presented in Table $1(21,22)$.

Table 1. Normal speckle derived strain values for left atrial phases (22)

\begin{tabular}{|l|c|}
\hline & Mean (95\% Cl) \\
\hline Reservoir & $39.4 \%(38.0-40.8 \%)$ \\
\hline Conduit & $23.0 \%(20.7-25.2 \%)$ \\
\hline Atrial Contraction & $17.4 \%(16.0-19.0 \%)$ \\
\hline
\end{tabular}

Systolic reservoir phase strain $(<23 \%)$ is more sensitive and specific for diagnosis of diastolic dysfunction compared to left atrial volume index or commonly used E/E' ratio $(23,24)$. 


\section{Steps for Myocardial Strain Measurement}

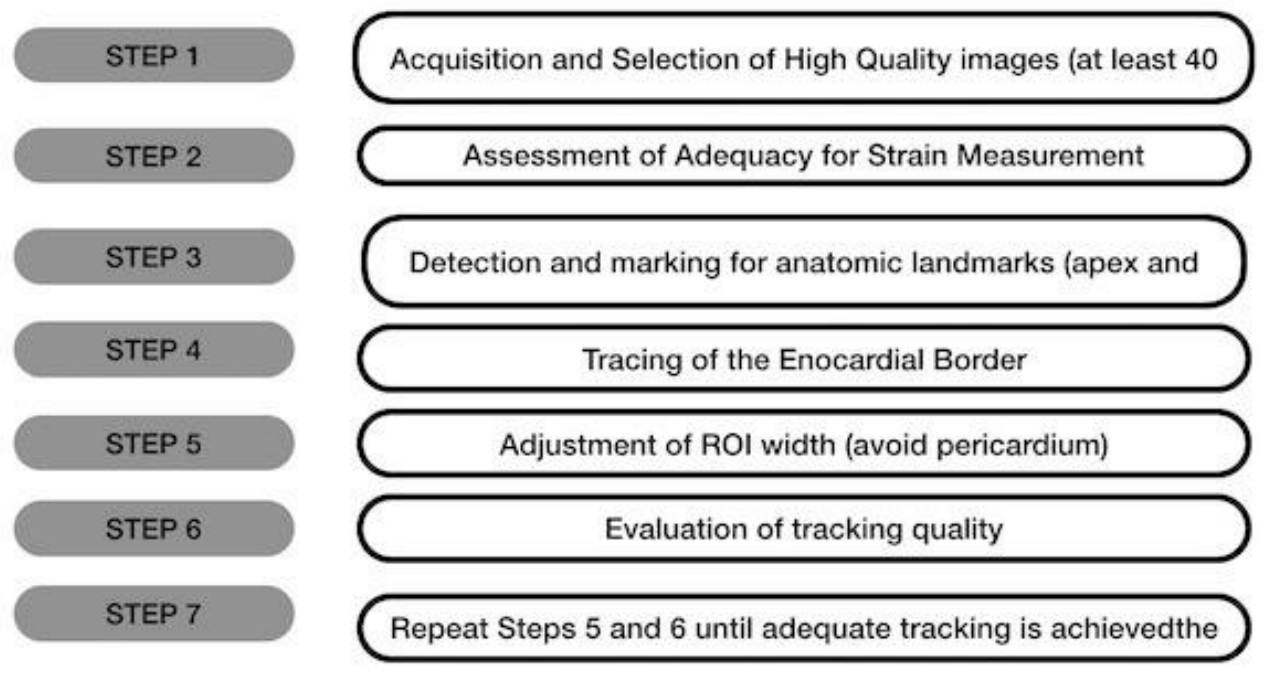

A)

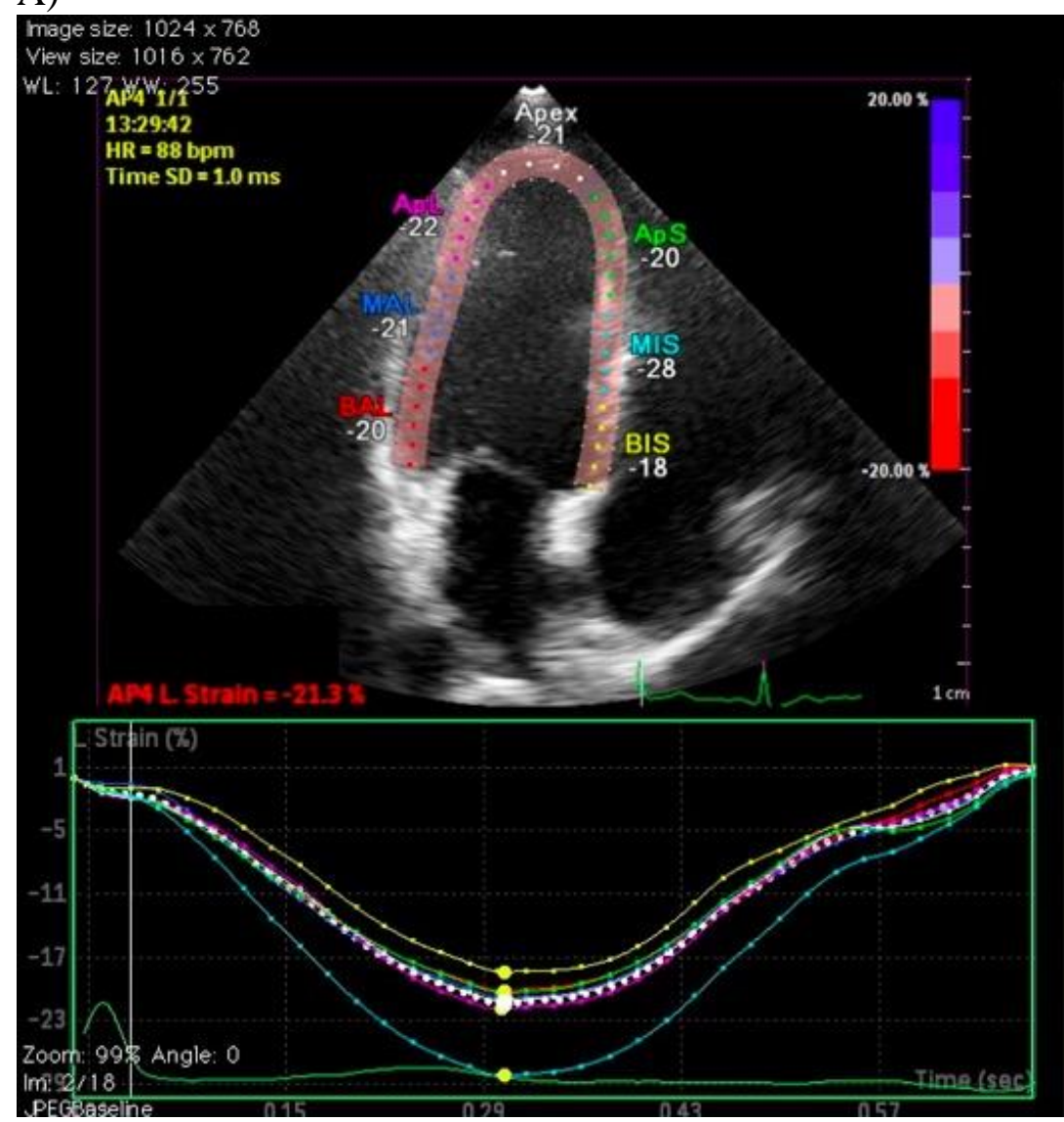

B)

Figure 2: a) How to calculate GLS b) A GLS measurement: An example from apical 4- chamber view (10) GLS - global longitudinal strain 

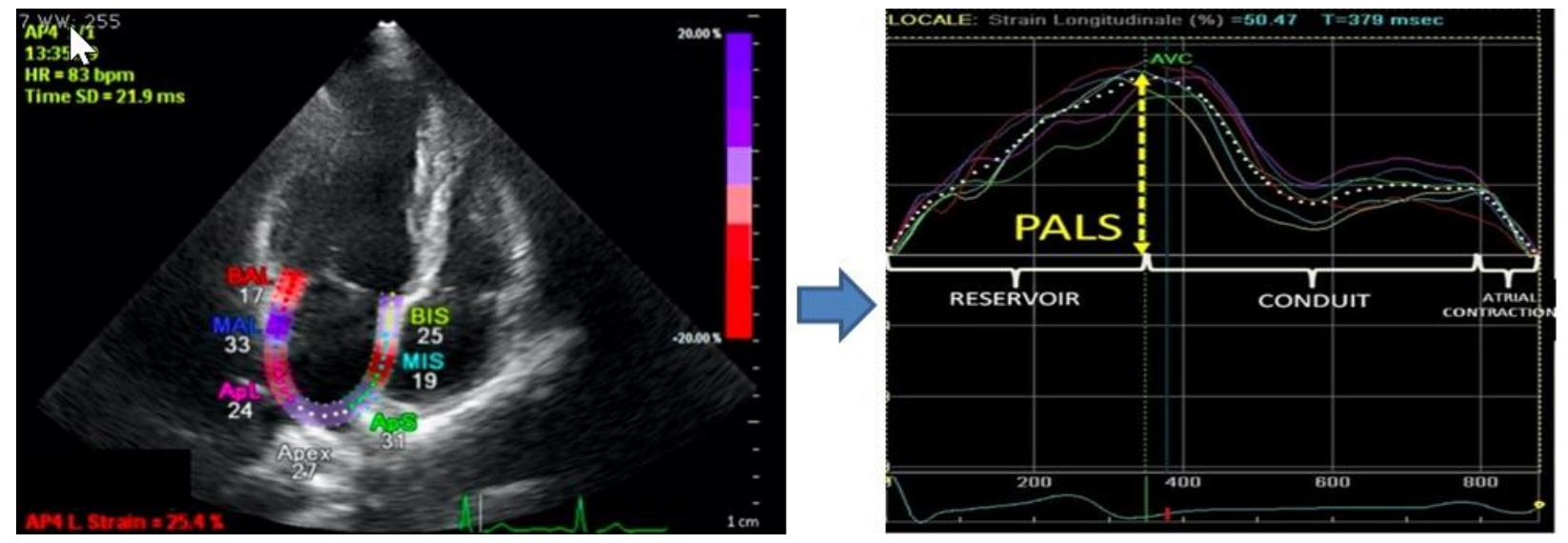

Figure 3. LA strain example (modified from reference 21) LA - left atrial, PALS- peak atrial longitudinal strain

Early changes as reflected by reduced strain has been found in hypertensive, diabetic , chronic kidney disease patients with normal left atrial volumes $(25,26)$. Patients with valvular heart disease also have diminished left atrial strain values. Severe mitral regurgitation patients with left atrial systolic strain less than or equal to $24 \%$ have worse survival regardless of symptom status (27). Similarly a decreased left atrial strain value in a patient with mitral or aortic stenosis is associated with worse cardiovascular outcomes and more frequent incident $\mathrm{AF}$ development $(28,29)$.

\section{Cardiac flow measurements}

$$
\text { Doppler echocardiography detects }
$$

unidirectional intracardiac flow velocities while it passes through a cardiac chamber or a valve. Recent technological innovations in imaging modalities have made it possible to assess multidirectional intracardiac blood flow in vivo.

Intracardiac blood flow is constrained by the shape of cardiac chambers and aligns with longitudinal filling-emptying mechanism. Asymmetrical ventricular shape causes formation of vortices during cardiac cycle within cardiac chambers (30). Better understanding of dynamic interaction between intracardiac blood flow and myocardial tissue deformation brings new opportunities for early diagnosis of diseases affecting heart, and by doing so, pave the way the prevention or the slowing of disease's progression (31).

\section{How Cardiac Vortex Develops}

In a tubular structure such as vessel, fluid layers at the center of the blood flow move faster compared to peripheral layers located at close vicinity of vessel wall due to friction. When blood flow abruptly enters a large chamber such as atrium or ventricle, there is a tendency for the peripheral layers of blood to spin away from the central jet (vorticity) (Fig. 4). Vorticity can cause the formation of vortex described as swirling motion spinning around a virtual central axis.

Venous blood flows from superior and inferior vena cava to the right atrium do not collide with each other. The orientation of right atrial blood flow favors the passage through tricuspid valve. Left atrial blood flow from pulmonary veins is also directed toward mitral valve (30). Both ventricles have diastolic blood flow oriented to their respective outflow regions, which provide a better efficiency for systolic ejection (30) (Fig. $5)$.

\section{Intracardiac Flow Imaging Methods}

Phase-contrast MRI (4D flow MRI) is the preferred method for intracardiac flow imaging. Echocardiography stands as an alternative platform with its lower cost, ready availability and shorter post processing time. Color-Doppler-based vector flow mapping (VFM) and particle image velocimetry with contrast use (Echo-PIV) have been developed for visualizing the intracardiac flow. 


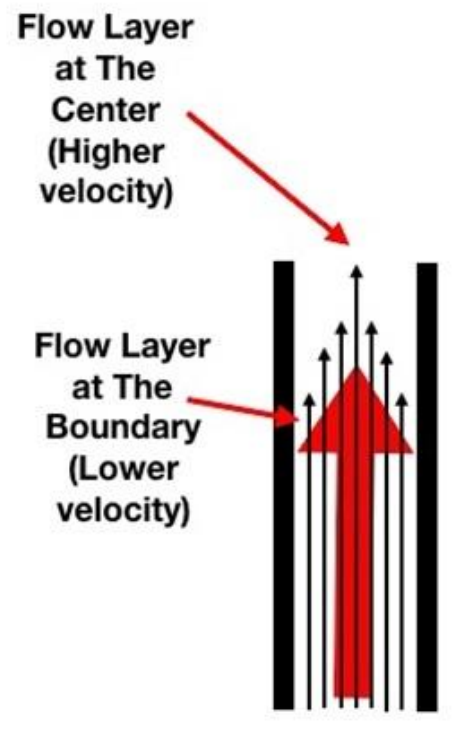

(a)

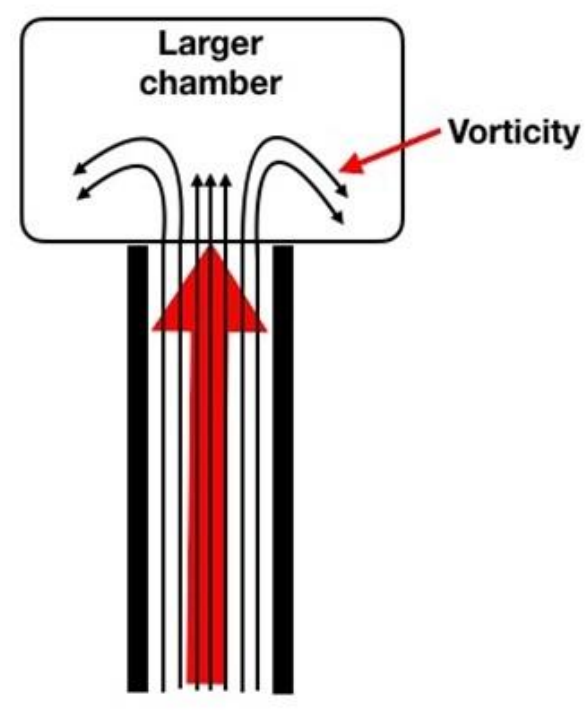

(b)

Figure 4. (a) Blood flow within the vessel (b) creation of vorticity when blood flow enters into larger chamber

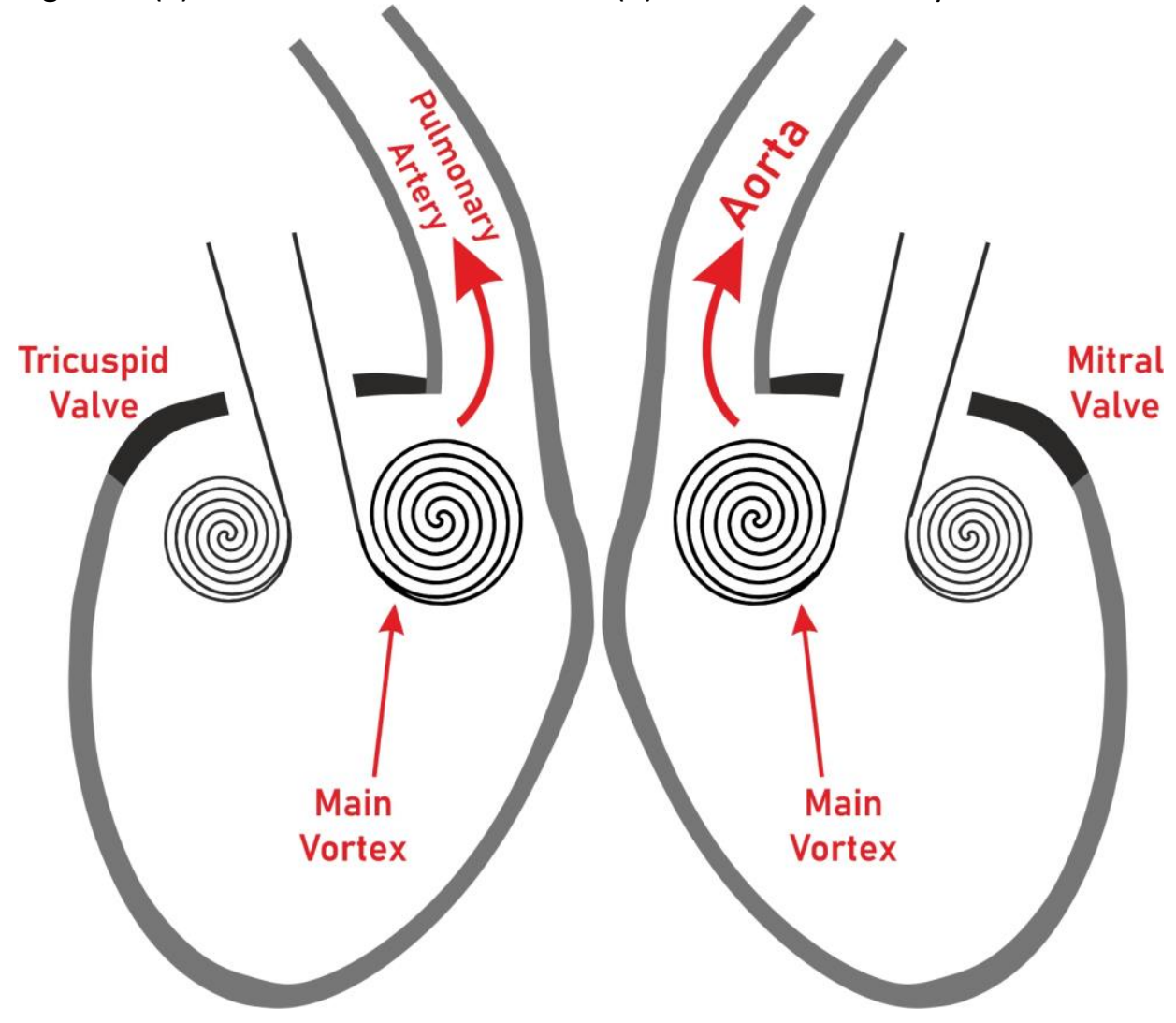

Figure 5. Vortex formation in right and left ventricles facilitating blood flow through their outflow regions. 


\section{a) Color-Doppler based vector flow mapping}

Color-Doppler collects information about unidirectional flow along the axial axis of ultrasound beam in an angle dependent way. VFM solves angle dependency with echo-dynamography based mathematical calculations (32). VFM creates vortical and nonvortical flow vectors from the measured axial velocities (parallel to the ultrasound beam) and the estimated radial velocities (perpendicular to the former ones) (33) (Fig. 6 (34)).

\section{b) Echocardiographic particle image velocimetry}

The motion patterns of contrast agent particles tracked ultrasonographically on a frame-by-frame basis in this technique.
The information about flow direction and velocity are obtained from the analyzed region such as left ventricle (35). Tracking of high velocity particles is limited by the need for very high frame rates, which restricts EchoPIV's clinical use and future development. Comparison of VFM and Echo-PIV methods is provided in Table 2 $(32,34)$.

Echo-PIV or VFM derived parameters (Vortex Depth, Vortex location, Vortex intensity, Vortex formation time etc.) have been used for the analysis of left ventricular, left atrial and right ventricular functions (34) (Fig. 7).

Table 2. Characteristics of color-Doppler-based vector flow mapping (VFM) and particle image velocimetry with contrast use (Echo-PIV) $(32,34)$

\begin{tabular}{|c|c|c|}
\hline & Echo-PIV & Color Doppler VFM \\
\hline Signal Source & Tracking of contrast microbubbles & Color Doppler based flow mapping \\
\hline Spatial Resolution & $\begin{array}{l}\text { Good spatial resolution in 2D, } \\
\text { limited 3D }\end{array}$ & Good spatial resolution in 2D and 3D \\
\hline Temporal Resolution & $\begin{array}{l}\text { High temporal resolution (4-20 } \\
\text { ms) }\end{array}$ & $\begin{array}{l}\text { Good temporal resolution in 2D }(4-20 \mathrm{~ms}) \text {, } \\
\text { relatively low in 3D }\end{array}$ \\
\hline 3D Coverage of All velocities & $\begin{array}{l}\text { in-plane components represented } \\
\text { but not the through- plane }\end{array}$ & $\begin{array}{l}\text { Only the } 1 \text { component directed to or from the } \\
\text { transducer is currently measurable clinically }\end{array}$ \\
\hline Scan Time & $\begin{array}{l}\text { Both scan time and offline } \\
\text { analysis can be done over few } \\
\text { heartbeats in minutes }\end{array}$ & Rapid scan times, real-time visualization \\
\hline Accuracy & $\begin{array}{c}\text { Good Low-velocity accuracy } \\
\text { Underestimated High-velocity } \\
\text { accuracy }\end{array}$ & $\begin{array}{c}\text { Underestimated Low-velocity accuracy } \\
\text { High-velocity accuracy resolved with optimal } \\
\text { aliasing velocity }\end{array}$ \\
\hline Advantages & $\begin{array}{l}\text { Bedside, lower cost, short process } \\
\text { time } \\
\text { Accurate visualized vortex } \\
\text { Validated quantitative parameters }\end{array}$ & $\begin{array}{l}\text { Bedside, lower cost, short process time } \\
\text { Do not require contrast microbubbles }\end{array}$ \\
\hline Limitations & $\begin{array}{l}\text { Need contrast agent } \\
\text { Need higher frame rate } \\
\text { Acoustic windows }\end{array}$ & $\begin{array}{l}\text { Lacking validated parameters } \\
\text { Need manual de-aliasing } \\
\text { Lower temporal resolution }\end{array}$ \\
\hline
\end{tabular}




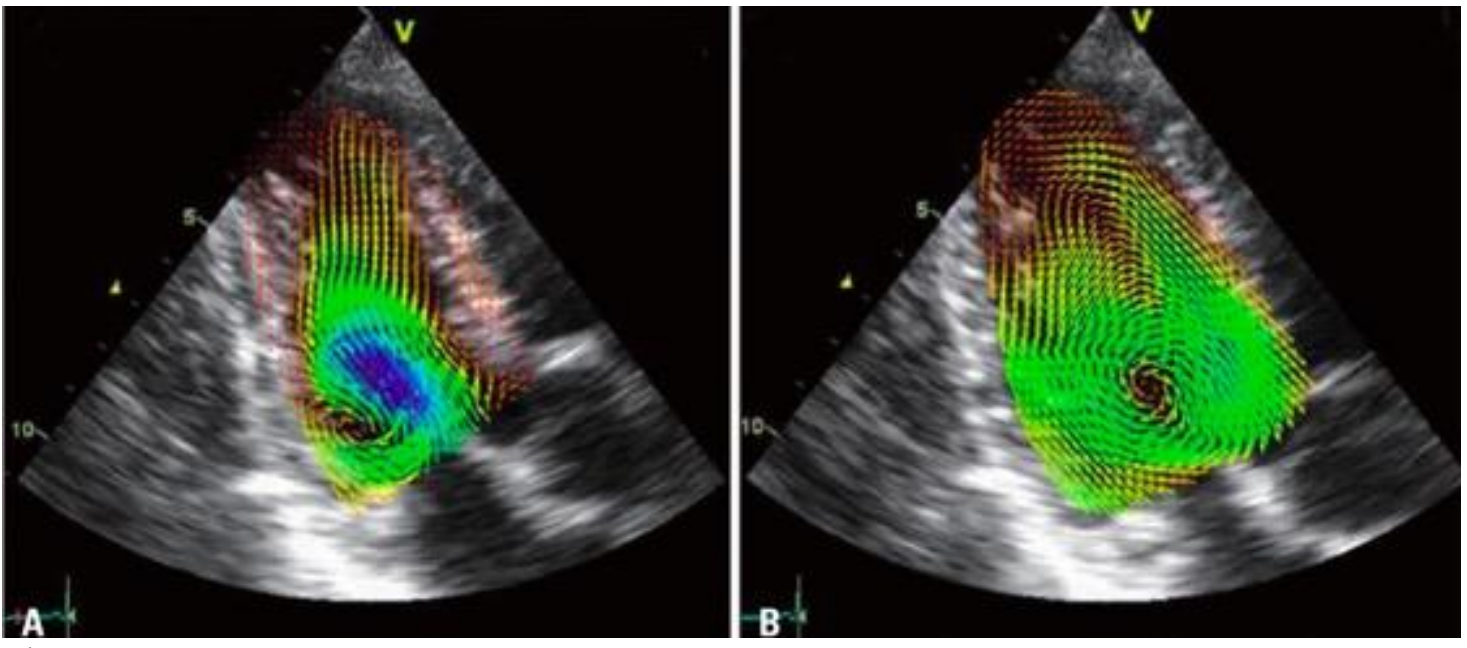

Figure 6. Examples of blood velocity mapping in a normal left ventricle overlaid on a sequence of anatomical B-mode apical long-axis images during early diastole (A), isovolumic contraction (B) (34).
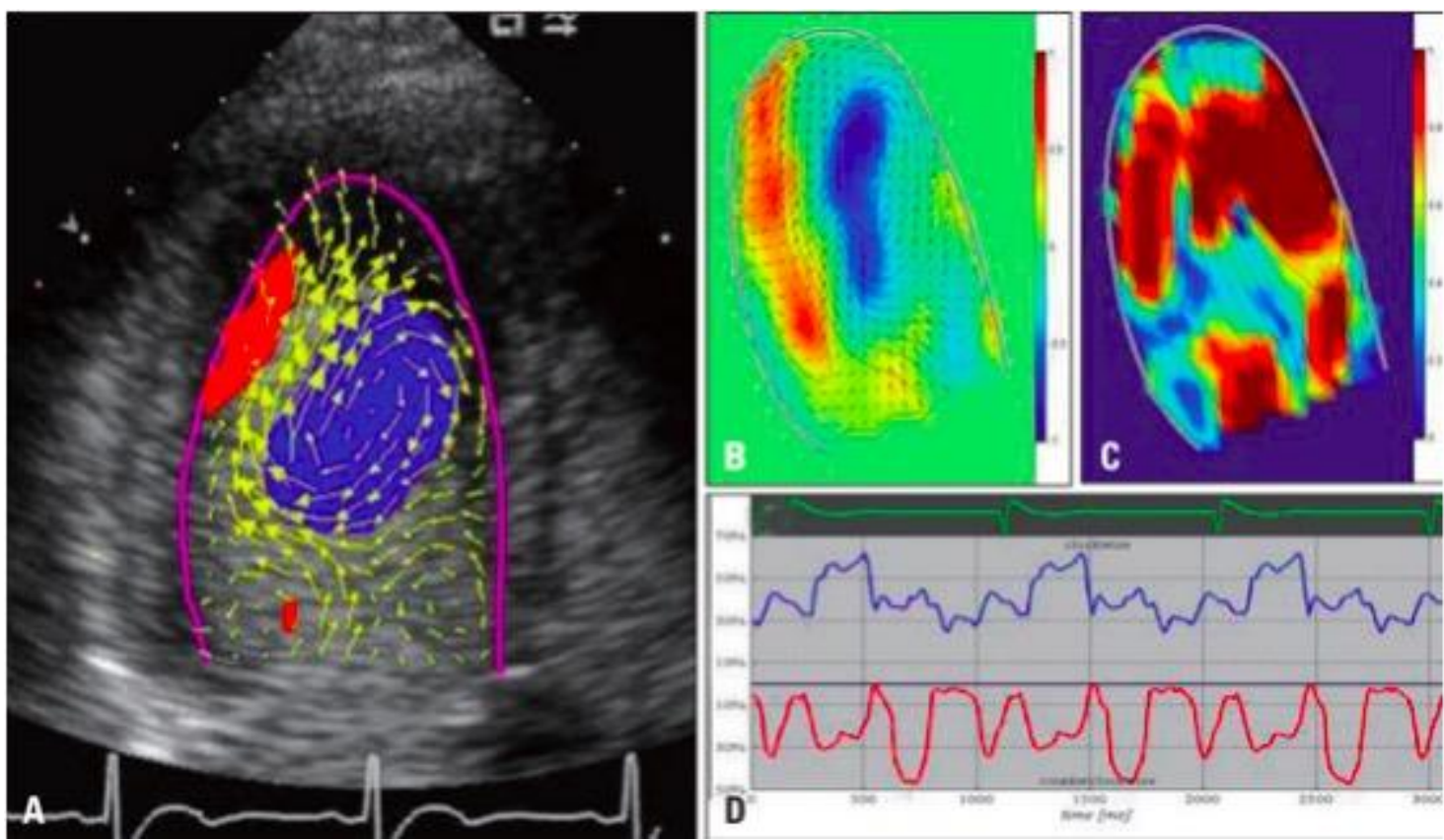

Figure 7. Example of left ventricular vortex flow analyzed by contrast echocardiography using particle image velocimetry method. The echo freeze frames represent the velocity vector on the scan-plane, superimposed to the reconstructed Doppler representation (A). Parametric representations of steady streaming field (B), pulsatile strength field (C) and vortex size change throughout the cardiac cycle (D) (34). 


\section{c) Speckle tracking and flow imaging}

Intracardiac blood flow and myocardial deformation intertwine with each other. Their mutual relation creates intraventricular pressure gradients (IVPG). Endocardial motion detected by speckle tracking has been used for estimating flow forces (IVPGs) within cardiac chambers $(36,37)$. This method is relatively new and more research is needed for better determination of its role in intracardiac flow imaging.

\section{Automated 3D echocardiographic left ventricular volume measurement}

An accurate calculation of ejection fraction has paramount importance in various disease states including but not restricted to heart failure and valvular heart disease. Visual assessment has been most frequently used method for EF determination with a questionable reliability. M-Mode derived EF calculation is almost completely abandoned and 2D based methods such as biplane disk summation or area-length are recommended in the chamber quantification guideline with their inherent property of underestimating true volumes (38).

$3 \mathrm{D}$ volume images can be captured in multiple heartbeats or in a recently introduced single heartbeat. This technique is free of any geometric assumption and yields more accurate volume values due to absence of the foreshortened images. 3D volume analysis is preferred over 2D volume analysis due to its better accuracy and reproducibility (38). It results in lower diastolic and systolic volumes compared to gold standard cardiac magnetic resonance imaging derived values but it is still more accurate when compared to $2 \mathrm{D}$ volume values (39). Interestingly, LVEF values are almost same among these modalities and can be used interchangeably (39).

The single beat 3D image acquisition provides similar accuracy for volumetric data and EF values compared to the multi beat method (40). It may also lessen stitching artifacts usually seen with irregular heart rhythms and obviate the need for prolonged breath holds as required in multi beat acquisition (41).

Adequate image quality directly affects the accuracy of any 3D volume analysis method. Lower spatial or temporal resolutions are major drawbacks for the technique. Full volume multi beat acquisition provides best solution for this problem but finding an ideal patient with good image quality, regular rhythm, satisfactory breath hold for optimal 3D image analysis is not possible every time. Moreover, single beat acquisition comes with even lower temporal and spatial resolution compared to multi beat method (40).

Both multi-beat and single-beat 3D datasets needs manual adjustments. The echocardiographer has to select appropriate imaging views, mark anatomical landmarks (mitral annulus, apex) and adjust ROI width and contours. These tasks are time-consuming and cause intolerable delays in a busy echo laboratory.

Semi-automatic software from various vendors such as TomTec 4D LV-Analysis (C) software (TomTec Imaging Systems), Philips QLab 3DQ-Advanced (Philips Healthcare) and GE 4D LVQ tool in the EchoPAC (GE Vingmed Ultrasound) have been used successfully in echo labs around the world but they still need manual corrections. Nevertheless, these semiautomatic programs have shorter analysis time with a favorable accuracy compared to manual method (42).

A fully automated 3D EF measurement completely eliminates any user input. Two vendors for this purpose are available: Siemens ultrasound eSie LV ATM tool integrated to ACUSON SC2000 PRIME (Siemens Healthcare) workplace and Philips HeartModel algorithm in the Philips EPIQ 7 machine. In a 3D volume dataset the software first identifies LV end-diastole (ECG gating) and then determines the global cardiac shape orientation. Inner (blood-tissue interface) and outer (compacted myocardium) borders are automatically detected (43) (Figure 8). LV end-systole is selected at the smallest left ventricular cavity. Preliminary end-systolic and end-diastolic LV and LA shapes are then built by using automatic endocardial surface detection. These created shapes are compared with a database containing various models from patients with different ventricular-atrial shapes and pathologies. Finally, the software matches most appropriate model with the patient's LV volume being analyzed. Endocardial border correction can be used when deemed necessary by the operator. 


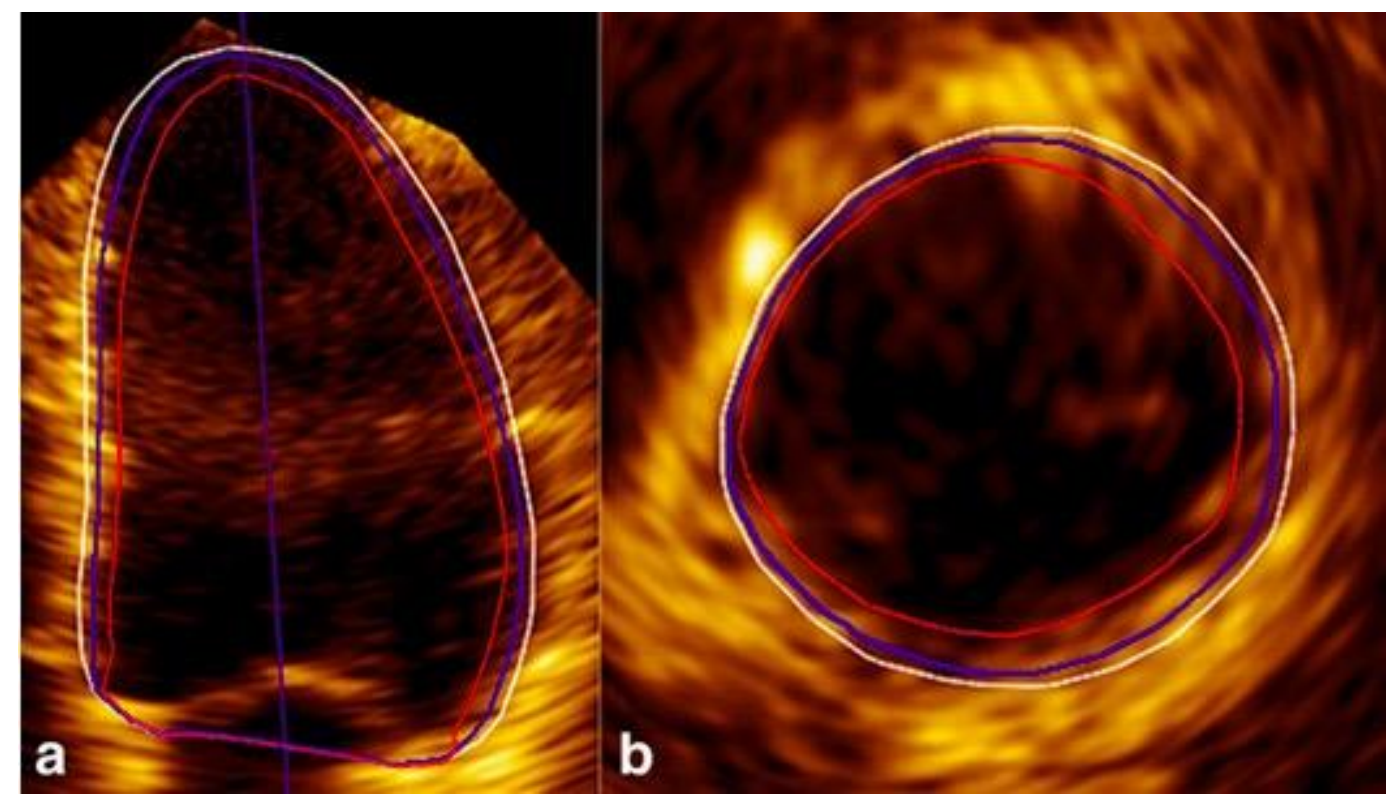

Figure 8. LV apical 4-chamber view (a) and basal short-axis view (b) in ED showed that the Heartmodel software detected the inner (red line) and outer extents of the myocardial tissue (white line). The LV endocardial border (blue line) is between them (43)

LV-left ventricle

Medvedofsky et al showed that automatic 3D EF analysis was not possible $10 \%$ of the patients (44). Poor image quality ( $24 \%$ of patients) is also associated with suboptimal agreement with manual 3D volume measurement. Automatic analysis had a very good agreement for the manual analysis in the remainder $66 \%$ of patients. A multicenter 3D automatic left ventricular volume analysis study with Philips HeartModel reported that automatic analysis had nearexcellent correlations with manual 3D volume analysis $(r=0.97,0.97$, and 0.96 for LV end-diastolic (EDV), LV end-systolic (ESV), and left atrial volume (LAV), respectively), while that for LV EF was lower $(r=0.88)$ (45). 3D automatic analysis underestimated left ventricular volumes $(-14 \pm 20 \mathrm{ml}$ for LVEDV, $-6 \pm 20 \mathrm{ml}$ for LVESV, and $-9 \pm 10 \mathrm{ml}$ for LAV), and LVEF $(-2 \pm 7 \%)(45)$. Authors of the study reached a conclusion that automated volumetric analysis of left-heart chambers is an accurate and robust alternative to conventional manual 3D methodology. This technique may contribute towards full integration of 3DE quantification into clinical routine, when such algorithms become universally available.

\section{Conclusion}

Echocardiography is an indispensable diagnostic test for any cardiologist. With modern echocardiography machines we can easily perform $\mathrm{M}$ mode or 2D dimension or volume measurements within seconds. We are prone to forget that current easy-toperform echocardiographic methods are novel research tools in the past. Myocardial deformation imaging has already taken its place in echo lab but intracardiac multidimensional flow determination and automated 3D volume analysis can be seen as immature tools for today's clinician. 3D automatic analysis has very strong potential to be incorporated into Artificial Intelligence Systems. If this task is accomplished, population level 3D echocardiographic volume data will be available for big data mining that result in unforeseen clinical solutions for different cardiac diseases. In the near future, we will be witnessing these new methods to be available in tomorrow's echocardiography machines. 
Peer-review: internal

Conflict of interest: none to declare

Authorship: O.B., E.P.O., Y.S., T.K.A. equally contributed to preparation of manuscript

Acknowledgement and funding: none to declare

\section{References}

1. Edler I, Lindström K. The history of echocardiography. Ultrasound Med Biol 2004; 30: 1565-644.

2. Konstam MA, Abboud FM. Ejection fraction: misunderstood and overrated (changing the paradigm in categorizing heart failure). Circulation 2017; 135:7179.

3. Urheim S, Edvardsen T, Torp H, Angelsen B, Smiseth OA. Myocardial strain by Doppler echocardiography. Validation of a new method to quantify regional myocardial function. Circulation 2000; 102: 1158-64.

4. Collier P, Phelan D, Klein A. A Test in context: myocardial strain measured by speckle-tracking echocardiography. J Am Coll Cardiol 2017; 69: 1043-56.

5. Amundsen $\mathrm{BH}$, Helle-Valle $\mathrm{T}$, Edvardsen $\mathrm{T}$, Torp $\mathrm{H}$, Crosby J, Lyseggen E et al. Noninvasive myocardial strain measurement by speckle tracking echocardiography: validation against sonomicrometry and tagged magnetic resonance imaging. J Am Coll Cardiol 2006; 47: 789-93.

6. Biering-Sorensen $T$, Hoffmann $S$, Mogelvang $R$, Zeeberg Iversen A, Galatius S, Fritz-Hansen $T$ et al. Myocardial strain analysis by 2-dimensional speckle tracking echocardiography improves diagnostics of coronary artery stenosis in stable angina pectoris. Circ Cardiovasc Imaging 2014; 7: 58-65.

7. Liu JH, Chen Y, Yuen M, Zhen Z, Chan CW, Lam KS et al. Incremental prognostic value of global longitudinal strain in patients with type 2 diabetes mellitus. Cardiovasc Diabetol 2016;15: 22.

8. Ross Agner BF, Katz MG, Williams ZR, Dixen U, Jensen $\mathrm{GB}$, Schwarz KQ. Left ventricular systolic function assessed by global longitudinal strain is impaired in atrial fibrillation compared to sinus rhythm. J Atr Fibrillation 2017; 10: 1437.

9. Negishi T, Negishi K, Thavendiranathan P, Cho GY, Popescu BA, Vinereanu D et al. Effect of Experience and Training on the Concordance and Precision of Strain Measurements. JACC Cardiovasc Imaging 2017; 10: 51822.
10. Negishi K, Negishi T, Kurosawa K, Hristova K, Popescu BA, Vinereanu $D$ et al. Practical guidance in echocardiographic assessment of global longitudinal strain. JACC Cardiovasc Imaging 2015; 8: 489-92.

11. Yingchoncharoen T, Agarwal S, Popović ZB, Marwick $\mathrm{TH}$. Normal ranges of left ventricular strain: a metaanalysis. J Am Soc Echocardiogr 2013; 26: 185-91.

12. Lang RM, Badano LP, Mor-Avi V, Afilalo J, Armstrong $A$, Ernande $L$ et al. Recommendations for cardiac chamber quantification by echocardiography in adults: an update from the American Society of Echocardiography and the European Association of Cardiovascular Imaging. Eur Heart J Cardiovasc Imaging 2015; 16: 233-70.

13. Farsalinos KE, Daraban AM, Ünlü $S$, Thomas JD, Badano LP, Voigt JU. Head-to-head comparison of global longitudinal strain measurements among nine different vendors: The EACVI/ASE inter-vendor comparison study. J Am Soc Echocardiogr. 2015;28:1171-1181, e2.

14. Plana JC, Galderisi M, Barac A, Ewer MS, Ky B, Scherrer-Crosbie $M$ et al. Expert consensus for multimodality imaging evaluation of adult patients during and after cancer therapy: a report from the American Society of Echocardiography and the European Association of Cardiovascular Imaging. J Am Soc Echocardiogr 2014; 27:911-39.

15. Jensen BV, Skovsgaard T, Nielsen SL. Functional monitoring of anthracycline cardiotoxicity: a prospective, blinded, long-term observational study of outcome in 120 patients. Ann Oncol 2002; 13: 699-709. 16. Plana JC, Thavendiranathan P, Bucciarelli-Ducci C, Lancellotti P. Multi-modality imaging in the assessment of cardiovascular toxicity in the cancer patient. JACC Cardiovasc Imaging 2018; 11: 1173-86.

17. Narayan HK, Finkelman B, French B, Plappert T, Hyman D, Smith AM et al. Detailed echocardiographic phenotyping in breast cancer patients: associations with ejection fraction decline, recovery, and heart failure symptoms over 3 years of follow-up. Circulation. 2017; 135: 1397-412.

18. Negishi K, Negishi T, Hare JL, Haluska BA, Plana JC, Marwick TH. Independent and incremental value of deformation indices for prediction of trastuzumabinduced cardiotoxicity. J Am Soc Echocardiogr 2013; 26: 493-8. 
19. Negishi T, Negishi K. Echocardiographic evaluation of cardiac function after cancer chemotherapy. J Echocardiogr 2018; 16: 20-7.

20. Badano LP, Kolias TJ, Muraru D, Abraham TP, Aurigemma G, Edvardsen T et al. Standardization of left atrial, right ventricular, and right atrial deformation imaging using two-dimensional speckle tracking echocardiography: a consensus document of the EACVI/ASE/Industry Task Force to standardize deformation imaging. Eur Heart J Cardiovasc Imaging 2018; 19: 591-600.

21. Cameli M, Lisi M, Mondillo S, Padeletti M, Ballo P, Tsioulpas $C$, et al. Left atrial longitudinal strain by speckle tracking echocardiography correlates well with left ventricular filling pressures in patients with heart failure. Cardiovasc Ultrasound 2010. doi:10.1186/14767120-8-14.

22. Pathan F, D'Elia N, Nolan MT, Marwick TH, Negishi K. Normal ranges of left atrial strain by speckle-tracking echocardiography: a systematic review and metaanalysis. J Am Soc Echocardiogr 2017; 30: 59-70.e8.

23. Morris DA, Belyavskiy E, Aravind-Kumar R, Kropf M, Frydas $A$, Braunauer $K$ et al. Potential usefulness and clinical relevance of adding left atrial strain to left atrial volume index in the detection of left ventricular diastolic dysfunction. JACC Cardiovasc Imaging 2018; 11: 1405-15.

24. Cameli M, Sparla S, Losito M, Righini FM, Menci D, Lisi $\mathrm{M}$ et al. Correlation of left atrial strain and Doppler measurements with invasive measurement of left ventricular end-diastolic pressure in patients stratified for different values of ejection fraction. Echocardiography 2016; 33: 398-405.

25. Mondillo S, Cameli M, Caputo ML, Lisi M, Palmerini $\mathrm{E}$, Padeletti $\mathrm{M}$ et al. Early detection of left atrial strain abnormalities by speckle-tracking in hypertensive and diabetic patients with normal left atrial size. J Am Soc Echocardiogr 2011; 24: 898-908.

26. Ohara Y, Yoshimura Y, Fukuoka Y, Matsumi H, Miyaji $T$, Sahara $S$ et al. Early detection of left atrial strain abnormalities by speckle-tracking in patients with chronic kidney disease and normal left atrial size. Eur Heart J 2013; 34: P2914.
27. Debonnaire P, Leong DP, Witkowski TG, Al Amri I, Joyce $E$, Katsanos $S$ et al. Left atrial function by twodimensional speckle-tracking echocardiography in patients with severe organic mitral regurgitation: association with guidelines-based surgical indication and postoperative (long-term) survival. J Am Soc Echocardiogr 2013; 26: 1053-62.

28. Ancona R, Comenale Pinto S, Caso P, Di Salvo G, Severino S, D'Andrea $A$ et al. Two-dimensional atrial systolic strain imaging predicts atrial fibrillation at 4year follow-up in asymptomatic rheumatic mitral stenosis. J Am Soc Echocardiogr 2013; 26: 270-7.

29. Galli E, Fournet $M$, Chabanne $C$, Lelong $B$, Leguerrier $A$, Flecher $E$ et al. Prognostic value of left atrial reservoir function in patients with severe aortic stenosis: a 2D speckle-tracking echocardiographic study. Eur Heart J Cardiovasc Imaging. 2016; 17: 533-41.

30. Kilner PJ, Yang G-Z, Wilkes AJ, Mohiaddin RH, Firmin $\mathrm{DN}$, Yacoub $\mathrm{MH}$. Asymmetric redirection of flow through the heart. Nature 2000; 404: 759.

31. Pedrizzetti G, La Canna G, Alfieri O, Tonti G. The vortex-an early predictor of cardiovascular outcome. Nature Reviews Cardiology 2014;11: 545.

32. Sengupta PP, Pedrizzetti G, Kilner PJ, Kheradvar A, Ebbers $\mathrm{T}$, Tonti $\mathrm{G}$ et al. Emerging trends in $\mathrm{CV}$ flow visualization. JACC: Cardiovascular Imaging. 2012; 5: 305-16.

33. Ohtsuki S, Tanaka M. The flow velocity distribution from the Doppler information on a plane in threedimensional flow. J Visualization 2006; 9: 69-82.

34. Hong G-R, Kim M, Pedrizzetti G, Vannan MA. Current clinical application of intracardiac flow analysis using echocardiography. J Cardiovasc Ultrasound 2013; 21: 155-62.

35. Hong G-R, Pedrizzetti G, Tonti G, Li P, Wei Z, Kim JK et al. Characterization and quantification of vortex flow in the human left ventricle by contrast echocardiography using vector particle image velocimetry. JACC: Cardiovascular Imaging 2008; 1: 705717.

36. Domenichini F, Pedrizzetti G. Hemodynamic forces in a model left ventricle. Physical Review Fluids 2016; 1 : 083201. 
37. Pedrizzetti G, Arvidsson PM, Töger J, Borgquist R, Domenichini $\mathrm{F}$, Arheden $\mathrm{H}$ et al. On estimating intraventricular hemodynamic forces from endocardial dynamics: a comparative study with 4D flow MRI. J Biomechanics 2017; 60: 203-10.

38. Lang RM, Badano LP, Mor-Avi V, Afilalo J, Armstrong $A$, Ernande L, et al. Recommendations for cardiac chamber quantification by echocardiography in adults:

an update from the American Society of Echocardiography and the European Association of Cardiovascular Imaging. Eur Heart J Cardiovasc Imaging. 2015; 16: 233-70.

39. Soliman OI, Kirschbaum SW, van Dalen BM, van der Zwaan HB, Mahdavian Delavary B, Vletter WB et al. Accuracy and reproducibility of quantitation of left ventricular function by real-time three-dimensional echocardiography versus cardiac magnetic resonance. Am J Cardiol 2008; 102: 778-83.

40. Macron L, Lim P, Bensaid A, Nahum J, Dussault C, Mitchell-Heggs $L$ et al. Single-beat versus multibeat realtime 3D echocardiography for assessing left ventricular volumes and ejection fraction: a comparison study with cardiac magnetic resonance. Circ Cardiovasc Imaging. 2010; 3: 450-5.
41. Ren B, Vletter WB, McGhie J, Soliman OI, Geleijnse ML. Single-beat real-time three-dimensional echocardiographic automated contour detection for quantification of left ventricular volumes and systolic function. Int J Cardiovasc Imaging 2014; 30: 287-94.

42. Leung KY, Bosch JG. Automated border detection in three-dimensional echocardiography: principles and promises. Eur J Echocardiogr 2010; 11: 97-108.

43. Sun L, Feng H, Ni L, Wang H, Gao D. Realization of fully automated quantification of left ventricular volumes and systolic function using transthoracic 3D echocardiography. Cardiovasc Ultrasound 2018; 16: 2.

44. Medvedofsky D, Mor-Avi V, Byku I, Singh A, Weinert $\mathrm{L}$, Yamat $\mathrm{M}$ et al. Three-dimensional echocardiographic automated quantification of left heart chamber volumes using an adaptive analytics algorithm: feasibility and impact of image quality in nonselected patients. J Am Soc Echocardiogr. 2017; 30: 879-85.

45. Medvedofsky D, Mor-Avi V, Amzulescu M, Fernández-Golfín C, Hinojar R, Monaghan MJ et al. Three-dimensional echocardiographic quantification of the left-heart chambers using an automated adaptive analytics algorithm: multicentre validation study. Eur Heart J-Cardiovasc Imaging 2017; 19: 47-58. 